\title{
Alpha-gal syndrome: the first report in Poland
}

\author{
MIRELLA BRZOZOWSKA ${ }^{1}$, NATALIA MOKRZYCKA ${ }^{2}$, GRZEGORZ POREBSKI ${ }^{3}$ \\ 'Department of Skin and Venereal Diseases, Clinical Hospital of the Pomeranian Medical University, Police, Poland \\ ${ }^{2}$ Jagiellonian University Medical College, Krakow, Poland \\ ${ }^{3}$ Department of Clinical and Environmental Allergology, Jagiellonian University Medical College, Krakow, Poland
}

\begin{abstract}
Alpha-gal syndrome is an immunoglobulin E (IgE)-dependent allergy to galactose- $\alpha$-1,3-galactose, resulting in a delayed anaphylactic reaction to red meat. The syndrome is causally linked to bites from ticks and associated with cross-reactivity to some drugs, e.g. cetuximab. Although cases of alpha-gal allergy have already been reported in a few European countries, to our best knowledge, no cases have been reported so far in Central-Eastern Europe. In the current report, we describe a case of alpha-gal syndrome diagnosed in Poland, to highlight the fact that it may occur in new geographic areas. Within three months, the described patient underwent five anaphylactic reactions with typical clinical manifestations. They developed a few hours after ingestion of red meat (pork, beef or mutton) and were preceded by tick bites. The level of specific IgE antibodies to alpha-gal reached $72.6 \mathrm{kAU} /$, whereas the levels of specific IgE antibodies to other food allergens were within the reference range. As the onset of symptoms in this syndrome is usually delayed, numerous cases may be identified as idiopathic anaphylaxis, while early diagnosis is indispensable to avoid potentially life-threatening complications.
\end{abstract}

Key words: allergy, anaphylaxis, alpha-gal syndrome.

(Cent Eur J Immunol 2021; 46 (3): 398-400)

\section{Introduction}

Alpha-gal syndrome is an immunoglobulin E (IgE)-dependent allergy to galactose- $\alpha$-1,3-galactose (alpha-gal), resulting in a delayed anaphylactic reaction to the consumption of red meat. The syndrome was first described in 2008 in the Southeastern United States and causally linked to bites from ticks [1]. Subsequently, there have been case reports in Australia, South Africa, Asia (Japan, South Korea), and in a few European countries (e.g., Sweden, Norway, France, Italy, Spain, Germany, Switzerland, and Austria) [1-6]. However, to our best knowledge, no cases have been reported so far in Central-Eastern Europe. Importantly, if undiagnosed, alpha-gal syndrome may result in severe hypersensitivity reactions to food and drugs [7]. Unfortunately, the syndrome can be easily overlooked due to relatively low awareness among healthcare professionals as well as some untypical clinical features. In the current paper, we describe a case of alpha-gal syndrome diagnosed in Poland and present some important clinical implications with the aim to draw the attention of clinicians to early diagnosis and management.

\section{Case report}

A 32-year-old man reported 5 anaphylactic reactions during the previous 3 months. The typical reaction involved abdominal pain and pruritic urticarial rash on the torso, forearms and lower limbs. One episode also induced shortness of breath and throat tightness. Symptoms occurred 3 to 6 hours after ingestion of red meat (pork, beef or mutton) and 2 to 3 hours after a meal of red meat accompanied by alcohol (in the amount of 2-3 standard alcohol units corresponding to one glass of red wine) or moderately intense physical exercise (cycling for $90 \mathrm{~min}-$ utes). Symptoms were independent of the type of alcohol consumed and appeared most quickly when both cofactors (alcohol and exercise) were present together. The severity of the reactions was usually determined by the amount of meat consumed. The patient experienced symptoms relief after taking an antihistamine drug (bilastine). He reported no symptoms after the consumption of chicken. He suffered 2 tick bites (probably from Ixodes ricinus). The first one occurred 2 weeks before the onset of anaphylactic symptoms. The patient's medical history was unremarkable. He did not report any previous allergic reactions, atopic conditions, episodes of anaphylaxis or comorbidities. The symptoms were not directly preceded by drug ingestion or insect stings.

The patient underwent a routine diagnostic workup for allergy, including alpha-gal-specific IgE antibodies and a panel of food allergens. Laboratory tests revealed significantly elevated level of IgE antibodies to alpha-gal (72.6 kAU/1, reference range $<0.35 \mathrm{kAU} / \mathrm{l}$ ), which was determined by a fluorometric enzyme immunoassay Immuno-

Correspondence: Dr. Grzegorz Porebski, Department of Clinical and Environmental Allergology, Jagiellonian University Medical College, Botaniczna 3, 31-503 Krakow, Poland, e-mail: g.porebski@uj.edu.pl Submitted: 26.08.2020; Accepted: 16.02.2021 
CAP (Thermo Fisher Scientific, Uppsala, Sweden). The levels of specific IgE antibodies to other food allergens were within the reference range (beef, crab, cod, celery, potato, carrot, tomato, kiwi, apricot, apple, almond, hazelnut, peanut, soybean, rice, wheat flour, rye flour, baking yeast, cow's milk, egg yolk, egg protein and cross-reactive carbohydrate determinants). Based on these findings, the diagnosis of alpha-gal syndrome was established and the patient was prescribed an emergency treatment. Additionally, he was instructed to avoid mammalian meat and other products containing alpha-gal. He has followed the recommendations and reported no further episodes to date.

\section{Discussion}

Galactose- $\alpha$-1,3-galactose is an oligosaccharide expressed on the cells of nonprimate mammals. In the course of evolution, Homo sapiens has lost the ability to synthesize alpha-gal; thus, the carbohydrate is immunogenic to humans [8]. Direct contact with the epitope leads to an increase in the production of alpha-gal-specific antibodies, and further exposure may trigger a strong immune response. According to recent studies, alpha-gal plays a major role in developing an anaphylactic reaction to red meat $[1,3]$. The symptoms are primarily seen after the consumption of meat from nonprimate mammals, such as beef, pork and lamb. Less frequently, they appear following the intake of products containing gelatin [9], while poultry and fish tend to be well tolerated [10]. Although the richest source of alpha-gal epitopes is pork kidney, any other product containing even small amounts of this antigen may likewise cause an allergic reaction [1, 7]. Symptoms of alpha-gal syndrome correspond to immediate IgE-mediated hypersensitivity (hives, nausea, rash, dyspnea, dizziness, abdominal pain, diarrhea, loss of consciousness and anaphylactic shock), but occur a few hours after the ingestion of the culprit food, namely, red meat [1].

Extended interviews with patients with a history of alpha-gal hypersensitivity revealed that previous exposure to a tick bite might have been the causative factor [4-6]. This association was confirmed by subsequent studies [7]. While Amblyomma americanum is the major tick species that causes morbidity in humans in the United States, Ixodes ricinus is the dominant species in Europe [1, 11]. Initially, alpha-gal epitopes in ticks were believed to originate from the gastrointestinal tract, from which they would be later transferred to the host's bloodstream during the bite. However, the latest studies have provided evidence for endogenous antigen synthesis in Ixodes ricinus [12].

According to recent reports, the hypersensitive response to alpha-gal might be triggered not only by red meat consumption but also by some drugs containing cross-reactive epitopes, such as cetuximab, an IgG monoclonal antibody drug used in the therapy of one of the most frequent malignancies - advanced colorectal cancer $[1,7$,
13]. Already the first infusion of cetuximab is sufficient for the development of anaphylaxis [1]. Other examples include the measles-mumps-rubella vaccine and zoster virus vaccine, as they have a significant amount of gelatin, which contains the alpha-gal epitope [14]. Also the gelatin cover of fenticonazole may be the source of epitopes, and thus the use of these intravaginal capsules may be associated with an allergic reaction [15]. Interestingly, as drug-induced symptoms develop rapidly, within minutes after the exposure, they are believed to be produced via the classic IgE-dependent mechanism of hypersensitivity. On the other hand, the delayed onset of a food-induced alpha-gal allergic reaction might potentially be explained by the difference in time needed for the epitope-rich molecules to reach the bloodstream. The digestion of alpha-gal-rich glycoproteins and glycolipids, together with their resorption in chylomicrons and very low-density lipoproteins, may take quite a long time [4]. Therefore, the first symptoms of red meat allergy may develop even several hours after consumption (ranging from 3 to 7 hours). It is possible that factors such as physical exercise, use of nonsteroidal antiinflammatory drugs and alcohol intake before red meat consumption can contribute to earlier onset [7]. On the other hand, direct intravenous infusion of cetuximab as well as application of fenticonazole capsules to a highly vascularized vaginal mucosa may reduce the reaction time even to a few minutes [15]. The above-mentioned cofactors (exercise, alcohol and nonsteroidal anti-inflammatory drugs) are associated with clinical manifestations of the alpha-gal syndrome. They enhance the allergic reaction and speed up the onset of symptoms in some patients. On the other hand, some individuals with $\operatorname{IgE}$ to alpha-gal may remain asymptomatic after exposure to the allergen in the absence of these cofactors [7, 16]. The main proposed mechanisms of these observations are an increase in blood circulation and in allergen absorption in the gastrointestinal tract induced by co-factors [16].

Alpha-gal allergy is diagnosed on the basis of typical signs and symptoms as well as the measurement of serum IgE antibody levels [1]. The alpha-gal specific IgE may be assessed as a separate measurement, which is nowadays available commercially in some specialized laboratories. It is also postulated to use new multiplexed methods such as the FABER test for this purpose [17]. The use of skin prick tests is not recommended, as they are not considered reliable [4]. Although various commercially available meat extracts are used in diagnostic workup, they may provide false-negative results [3]. Patients with this type of allergy are strongly advised to avoid exposure to any potential sources of alpha-gal. Thus, it may be necessary to eliminate red meat, gelatin-containing products, and often even dairy from the diet. Moreover, there may be some limitations to the use of drugs and vaccines $[1,7]$. It is also advisable to prescribe emergency treatment, including an epinephrine autoinjector, in case of sudden onset of severe symptoms. 


\section{Conclusions}

The aim of this report was to increase the awareness of alpha-gal syndrome among clinicians and to highlight the fact that it may occur in new geographic areas. As the onset of symptoms may be delayed, numerous cases may remain undiagnosed or may be identified as idiopathic anaphylaxis, while early diagnosis is indispensable to avoid potentially life-threatening complications.

\section{Acknowledgments}

This work was supported by a grant from the Faculty of Medicine, Jagiellonian University Medical College (N41/DBS/000324; to GP). The preliminary results were presented at the European Academy of Allergy and Clinical Immunology Digital Congress 2020 (June 6-8, 2020).

The authors declare no conflict of interest.

\section{References}

1. Platts-Mills TAE, Li RC, Keshavarz B, et al. (2020): Diagnosis and management of patients with the $\alpha$-gal syndrome. J Allergy Clin Immunol Pract 8: 15-23.e1.

2. Schmidle P, Reidenbach K, Kugler C, et al. (2019): Recall urticaria: a new clinical sign in the diagnosis of alpha-gal syndrome. J Allergy Clin Immunol Pract 7: 685-686.

3. Hodžić A, Mateos-Hernández L, de la Fuente J, Cabezas-Cruz A (2019): Delayed hypersensitivity reaction to mammalian galactose- $\alpha$-1,3-galactose ( $\alpha$-Gal) after repeated tick bites in a patient from France. Ticks Tick Borne Dis 10: 1057-1059.

4. Pisazka V, Duscher G, Hodžić A, et al. (2019): Alpha-gal allergy after a tick bite in Austria. Wien Klin Wochenschr 131: 385-388.

5. Arslan Lied G (2017): Red meat allergy induced by tick bites: a Norwegian case report. Eur Ann Allergy Clin Immunol 49: 186-188.

6. Calamari AM, Poppa M, Villalta D, Pravettoni V (2015): Alpha-gal anaphylaxis: the first case report in Italy. Eur Ann Allergy Clin Immunol 47: 161-162.

7. Hilger C, Fischer J, Wölbing F, Biedermann T (2019): Role and mechanism of galactose-alpha-1,3-galactose in the elicitation of delayed anaphylactic reactions to red meat. Curr Allergy Asthma Rep 19: 3.

8. Galili U, Shohet SB, Kobrin E, et al. (1988): Man, apes, and Old World monkeys differ from other mammals in the expression of alpha-galactosyl epitopes on nucleated cells. J Biol Chem 263: 17755-17762.

9. Lied GA, Lund KB, Storaas T (2019): Intraoperative anaphylaxis to gelatin-based hemostatic agents: a case report. J Asthma Allergy 12: 163-167.

10. Kollmann D, Nagl B, Ebner C, et al. (2017): The quantity and quality of $\alpha$-gal-specific antibodies differ in individuals with and without delayed red meat allergy. Allergy 72: 266-273.

11. Hamsten C, Starkhammar M, Tran TA, et al. (2013): Identification of galactose- $\alpha-1,3$-galactose in the gastrointestinal tract of the tick Ixodes ricinus; possible relationship with red meat allergy. Allergy 68: 549-552.
12. Cabezas-Cruz A, Espinosa PJ, Alberdi P, et al. (2018): Tick galactosyltransferases are involved in $\alpha$-Gal synthesis and play a role during Anaplasma phagocytophilum infection and Ixodes scapularis tick vector development. Sci Rep 8: 14224.

13. Kasztelan-Szczerbińska B, Cichoz-Lach H, Słomka M (2008): Colorectal cancer as a health care problem: evaluation of the current diagnostic options. Pol Arch Med Wewn 118: 224-227.

14. Stone CA Jr, Hemler JA, Commins SP, et al. (2017): Anaphylaxis after zoster vaccine: implicating alpha-gal allergy as a possible mechanism. J Allergy Clin Immunol 139: 17101713.e2.

15. Vidal C, Méndez-Brea P, López-Freire S, González-Vidal T (2016): Vaginal capsules: an unsuspected probable source of exposure to $\alpha$-gal. J Investig Allergol Clin Immunol 26: 388-389.

16. Cabezas-Cruz A, Hodžić A, Román-Carrasco P, et al. (2019) Environmental and molecular drivers of the $\alpha$-gal syndrome. Front Immunol 10: 1210.

17. Buczyłko K (2017): Zespół alfa-gal - nowe fakty kliniczne, nowe techniki diagnostyczne. Alergia 2: 36-38. 